\title{
Narrativas Digitais e Aprendizagem: um panorama a partir do ensino da saúde
}

\section{Digital Storytelling and Learning: an overview based on Health Education}

\section{Relatos Digitales y Aprendizaje: una visión general desde la enseñanza de la salud}

\author{
Maria Augusta Vasconcelos Palácio | augustapalacio@yahoo.com.br \\ Universidade Regional do Cariri, Departamento de Enfermagem. Crato, Ceará, Brasil. \\ Diana Ciannella | diciannella@yahoo.com.br \\ Universidade Federal do Rio de Janeiro, Núcleo de Tecnologia Educacional para a Saúde. Rio de Janeiro, Brasil. \\ Miriam Struchiner | miriamstru@gmail.com \\ Universidade Federal do Rio de Janeiro, Núcleo de Tecnologia Educacional para a Saúde. Rio de Janeiro, Brasil.
}

\section{Resumo}

A narrativa digital integra diferentes linguagens midiáticas com o potencial da narrativa de facilitar a construção de sentido e o compartilhamento de experiências individuais. No ensino da saúde é recorrente a discussão sobre a necessidade de mudança nas práticas pedagógicas tradicionais com a valorização das experiências dos educandos e a promoção da sua participação ativa e reflexiva. A discussão sobre a narrativa digital considera as ideias de Bruner sobre o valor educativo da narrativa e sua capacidade de organizar experiências, promover a reflexão e transmitir significados. Trata-se de uma revisão bibliográfica em periódicos internacionais sobre a contribuição da narrativa digital no ensino da saúde, incluindo somente artigos que apresentavam práticas pedagógicas sobre o tema. Os resultados revelam práticas com o uso da narrativa digital em diferentes contextos, envolvendo alunos em formação e profissionais de saúde. Os estudos compartilham a observação de que a narrativa digital possibilita a promoção da criatividade, criticidade e reflexão do estudante.

Palavras chave: narrativa digital; tecnologias de informação e comunicação; ensino da saúde; aprendizagem; revisão. 


\section{Abstract}

Digital storytelling integrates different digital media with narrative potential to facilitate individuals in sharing experiences and constructing meaning. In the Health Education is a recurring discussion the need for change in traditional teaching practices with the appreciation of the student's experiences and promoting active and reflective participation. The discussion about digital storytelling is based on Bruner's ideas about storytelling educational value and about its potential to organize experiences, to promote reflection, and to share meaning. This article presents a literature review about digital storytelling contribution to Health Education. The articles were selected in international journals, and the analysis included only those presenting pedagogical practices on the subject. The results show pedagogical practices with the use of digital storytelling in different contexts, involving students in training and health professionals. The studies share the observation that digital storytelling enables the promotion of creativity, critical skills and student reflection.

Keywords: digital storytelling; information and communication technologies; health education; learning; review.

\section{Resumen}

El relato o narrativa digital incorpora distintos medios con el potencial que tiene la narrativa de facilitar la construcción de sentido y el intercambio de experiencias individuales. En la enseñanza de la salud, es discusión recurrente la necesidad de un cambio en las prácticas tradicionales de enseñanza con la apreciación de las experiencias de los estudiantes y la promoción de su participación activa y reflexiva. La discusión sobre la narrativa digital considera las ideas de Bruner sobre el valor educativo vinculado al acto de narrar y por su capacidad de organizar experiencias y transmitir significados. Se presenta una revisión de la literatura sobre la contribución del relato digital en la enseñanza de la salud. Los artículos fueron seleccionados en periódicos internacionales, y el análisis incluyó solamente los que presentaban prácticas pedagógicas sobre el tema. Los resultados muestran prácticas con el uso de los relatos digitales en diferentes contextos, con estudiantes en formación y profesionales de la salud. Los estudios comparten la observación de que el relato digital permite la promoción de la creatividad, criticidad y la reflexión de los estudiantes.

Palabras clave: relato digital; narrativa digital; tecnologías de la información y comunicación; enseñanza de la salud; aprendizaje; revisión.

Contribuição dos autores: Maria Augusta Vasconcelos Palácio, Diana Ciannella e Miriam Struchiner participaram na concepção e redação do artigo.

Declaração de conflito de interesses: Não há conflitos de interesse

Fontes de financiamento: FAPERJ - Fundação Carlos Chagas Filho de Amparo à Pesquisa do Estado do Rio de Janeiro; CAPES - Coordenação de Aperfeiçoamento de Pessoal de Nível Superior e CNPq - Conselho Nacional de Desenvolvimento Científico e Tecnológico

Considerações éticas: Não se aplica

Agradecimento/Contribuições adicionais: Não houve

Histórico do artigo: Submetido: 10.abr.2016 | Aceito: 11.maio.2017 | Publicado: 30.jun.2017

Apresentação anterior: 0 artigo não foi apresentado anteriormente

Licença CC BY-NC atribuição não comercial. Com essa licença é permitido acessar, baixar (download), copiar, imprimir, compartilhar, reutilizar e distribuir os artigos, desde que para uso não comercial e com a citação da fonte, conferindo os devidos créditos de autoria e menção à Reciis. Nesses casos, nenhuma permissão é necessária por parte dos autores ou dos editores. 


\section{Introdução}

$\mathrm{O}$ ato de narrar acompanha todas as nossas ações e relações sociais e nos ajuda a dar sentido aos acontecimentos, uma vez que, ao narrarmos uma experiência, somos remetidos ao registro da memória sobre o cotidiano da vida social, ao específico do sujeito, ao coletivo de um grupo, aos significados que os sujeitos atribuem aos acontecimentos ${ }^{1}$. Dessa forma, a narrativa pode se constituir na construção de um pensamento crítico no sentido de tomar decisões e refletir sobre as formas de representação mais adequadas para transmitir melhor o que se deseja ${ }^{2}$.

Jerome Bruner ${ }^{3}$ descreve a narrativa como um modo de pensar, uma forma de organizar nossa experiência e, portanto, como um recurso no processo da educação. Bruner ${ }^{4}$ atribui valor educativo ao ato de narrar, por sua capacidade de organizar as experiências e transmitir significados. Para o autor, a narrativa assume algumas características que orientam a sua construção e compreensão, dentre elas: a sua organização em início, meio e fim, configurando uma estrutura de tempo determinada pela ocorrência dos eventos; as narrativas estão relacionadas a aspectos particulares, ou seja, contam experiências individuais, mas sua função reside no fato de permitir o compartilhamento dessa experiência; todas as ações têm razões implícitas e são motivadas por crenças, desejos, teorias e valores dos sujeitos; e, por fim, toda narrativa possui múltiplos significados, pois não há uma interpretação singular4.

Explorar a narrativa para acessar a experiência do aluno no processo de ensino-aprendizagem é, desta forma, um meio para o entendimento de como ele representa o conhecimento a partir da relação com o contexto de formação no qual está inserido. Além disso, esse movimento cria elementos para que o aluno possa refletir sobre suas vivências de aprendizagem. A reflexão nasce, portanto, da experiência, bem como da forma como esta é compartilhada. As discussões sobre a importância da experiência nas práticas educativas ganham uma maior dimensão a partir dos trabalhos de autores que reorganizaram a forma de olhar para o sujeito educando, dentre eles John Dewey (1859 - 1952), Paulo Freire (1921-1997) e Celèstin Freinet (1896 - 1966). Embora apresentem perspectivas diferentes, a obra desses autores é referência quanto ao valor que a experiência exerce no processo de aprendizagem.

Existem várias formas de produção de narrativa, oral, escrita e relatos multimídia, que mais recentemente, com o advento das tecnologias digitais de informação e comunicação (TDIC), têm sido utilizadas em diferentes contextos formativos. Com as novas mídias, criam-se possibilidades de representação em novos formatos de expressão e comunicação. A narrativa digital, deste modo, adquire um interesse educativo, uma vez que se configura como um meio para o aluno dar sentido ao que faz, aumentar a sua motivação em relação ao aprendizado e aproximar cada vez mais as TDIC dos currículos².

No ensino da saúde, há um movimento que defende a ressignificação da formação nessas profissões, como uma questão inadiável e alcançável a partir de propostas de mudanças relacionadas aos processos de formação, às relações entre os sujeitos envolvidos e ao conteúdo das disciplinas, bem como à reforma curricular dos cursos 5 . Trata-se de um movimento que, em grande parte, envolve a mudança no papel do aluno, para que este assuma seu protagonismo no processo educativo. Há, portanto, uma crescente tendência pela busca de métodos inovadores que envolvam uma prática pedagógica ética, crítica, reflexiva e transformadora, capaz de alcançar a formação do homem como um ser histórico, inscrito na dialética da ação-reflexão-ação ${ }^{6}$. Como promover a participação ativa do aluno nesse processo educativo é uma questão a ser discutida e problematizada no ensino da saúde. Entendemos a contribuição da narrativa digital por unir os elementos próprios da narrativa e sua relação com a educação e, ao mesmo tempo, o potencial das tecnologias digitais em diferentes contextos formativos.

A narrativa é geradora de mudanças na maneira como as pessoas compreendem suas próprias atitudes e as dos outros, pois na medida em que o sujeito consegue se ver no que ele produziu, na sua história apresentada sob diferentes formas, ele se torna capaz de teorizar a sua própria experiência ${ }^{7-8}$. No contexto 
do ensino da saúde, isso se torna relevante para pensar, por exemplo, a compreensão que o aluno tem sobre sua experiência, seja na relação profissional de saúde-paciente ou aluno-docente, e os significados que são construídos e reconstruídos a partir dela. A capacidade de apresentar a sua experiência e dar sentido aos fatos pode representar um movimento emancipatório para o sujeito, uma vez que ele aprende a produzir a sua formação, autodeterminando a sua trajetória7. No entanto, para que seja emancipatório é preciso que seja crítico, ou seja, que a narrativa não esteja dissociada de um movimento reflexivo.

Nessa perspectiva, a narrativa digital surge como um caminho para discutirmos um processo educativo que valorize as experiências dos sujeitos educandos e promova a sua participação ativa e reflexiva. Com o interesse em discutir a contribuição da narrativa digital no ensino da saúde, apresentamos neste artigo uma revisão bibliográfica em periódicos internacionais sobre esta temática. Orientamos nossa pesquisa pela seguinte pergunta: Como se caracterizam as práticas pedagógicas que incluíram a narrativa digital em contextos educativos no ensino da saúde?

\section{Fundamentação Teórica}

A narrativa, arte milenar de contar histórias, tem ganhado novos significados e usos com o advento das tecnologias digitais. Caracterizadas como narrativas digitais, digital storytelling ou relatos digitales, essas novas formas de representação da narrativa, assim como as tradicionais, guardam as mesmas caraterísticas de se constituírem a partir de um ponto de vista particular do indivíduo ou se relacionarem à apresentação sobre um tema específico $0^{9-10}$. Foi a partir de meados do século $\mathrm{XX}$, com o maior desenvolvimento das tecnologias, como o rádio, a televisão, o cinema e, mais recentemente, o computador e a internet; e aqui se destacam as ferramentas que nascem dessa geração, como os recursos da Web 2.o, que tem possibilitado a disseminação de diversos tipos de narrativa ${ }^{11}$.

A narrativa digital se constitui como uma interface de ensino e aprendizagem que envolve professores e alunos em atividades que favorecem a interação, motivação e criatividade, ajudando estes últimos a organizar suas ideias, fazer perguntas, expressar opiniões e, com isso, refletir sobre o processo educativo ${ }^{9-10}$. Desde o final da década de 1980, o Center for Digital Storytelling - CDS, uma organização sem fins lucrativos em Berkeley, Califórnia, tem fornecido treinamento e assistência para pessoas interessadas em criar e compartilhar suas narrativas pessoais, que se configuram como um tipo de narrativa digital ${ }^{10}$. Esse tipo de narrativa combina a arte de contar histórias com uma variedade de mídias, relacionadas diretamente ao uso das ferramentas tecnológicas, como o computador, dispositivos de captura de imagens e de áudio, bem como o uso de software para criação e edição das narrativas, um dos formatos mais utilizados para fins educacionais ${ }^{9-10}$.

De acordo com Jesus ${ }^{11}$, “a narrativa digital possibilita a utilização de diferentes sistemas de representação do conhecimento e de significação, incluindo formas verbais e não verbais” (p.51). Para Almeida e Valente', aplicativos da Web 2.o, como o Flash, o Movie Maker, os Blogs, entre outros tantos, ou até mesmo aplicativos convencionais para produção de apresentações, como o PowerPoint, podem ser utilizados para a produção de narrativas. A facilidade de manipulação dessas diferentes mídias permite que as pessoas sejam autoras, produtoras e disseminadoras de conhecimento ${ }^{1}$.

Um aspecto que chama atenção no estudo da narrativa digital está relacionado à avaliação desse tipo de produção. Explorar o que essa linguagem midiática oferece quando presente nas narrativas é um ponto que requer discussão. Em geral, quando são utilizadas em contextos de aprendizagem, o conteúdo da narrativa e a representação da experiência a partir de distintas linguagens, como imagens ou fotografias feitas pelos próprios alunos, vídeos ou gravações, são os elementos mais comumente analisados. O caráter multimodal dessas construções narrativas implica um olhar ampliado para os elementos que as compõem. Conforme destacam Hull e Nelson ${ }^{12}$, os aspectos que caracterizam uma composição multimodal, como se constituem as narrativas digitais, não são apenas um conjunto de diferentes elementos que estão justapostos em um 
mesmo espaço, como as imagens, o texto ou a música, mas como elementos que, juntos, podem ampliar o significado de um texto. Em outras palavras, "um texto multimodal pode criar um sistema diferente de significação, que transcende a contribuição coletiva de suas partes constituintes”"12 (p.225).

Dessa forma, cada elemento da narrativa digital merece uma atenção especial quanto às suas possibilidades. A imagem, por exemplo, fortemente presente nesse tipo de produção, se constitui um dos elementos fundamentais na narrativa digital, pois é ela que, em um primeiro momento, tem a capacidade de prender a atenção do público ${ }^{13}$. Para o autor, "as imagens têm uma capacidade especial de transmitir algo que as palavras não conseguem"13 (p.24). No estudo de Lourenço e Ramos ${ }^{14}$, os alunos, por meio de entrevistas, revelaram que haviam desenvolvido a capacidade de se comunicar pelas imagens e entender o seu valor na narrativa digital.

A imagem, enquanto mensagem visual composta por diferentes signos, equivale a uma linguagem muito valiosa e, dessa forma, importante instrumento de expressão e comunicação ${ }^{15}$. Muitas vezes, por meio de fotografias ou imagens capturadas em sítios na internet, os alunos poderão representar suas experiências de aprendizagem sem perder os significados atrelados a estas vivências. "Quer ela seja expressiva ou comunicativa, podemos admitir que uma imagem constitui sempre uma mensagem para o outro, mesmo quando este outro é o próprio autor da mensagem"15 (p.61). Deste modo, representa um importante caminho para um movimento reflexivo do aluno. Sobre esta relação do uso de imagens com a reflexão, é importante considerar que as fotografias, por exemplo, guardam a particularidade de ser um instrumento de resgate para a memória, uma vez que nos leva de volta a um momento específico no tempo e ajuda na reflexão retrospectiva ${ }^{16}$.

Outra importante linguagem que integra a narrativa digital, devido às suas possibilidades de uso, é o vídeo. No entanto, segundo Figueiredo ${ }^{13}$, este recurso muitas vezes é confundido com o produto final da narrativa digital. Isso acontece pois, nas suas origens, principalmente àquelas relacionadas à produção de narrativas pessoais, o vídeo é o instrumento pelo qual são apresentadas essas narrativas. Embora exista uma importante produção científica que mostra o vídeo como principal elemento para a construção da narrativa digital, há outras possibilidades de apresentação dessa narrativa, que podem inclusive requerer em algum momento, o uso de vídeos para ajudar a narrar determinados eventos. Neste sentido, corroboramos com Figueiredo ${ }^{13}$, ao destacar que o vídeo poderá ser apenas um dos elementos a integrar o projeto da narrativa digital, em conjunto com toda uma diversidade de elementos representados por imagens, áudio e texto.

O estudo sobre a narrativa digital em contextos educativos também está relacionado às oportunidades que esta metodologia de trabalho oferece ao que é denominado como literacia/letramento digital. Para Hack e Guedes ${ }^{17}$, a literacia digital se refere à habilidade para usar e compreender o uso das múltiplas tecnologias no cotidiano, de um modo geral, e em contextos educativos, de modo particular. Estes autores citam o trabalho de Ohler (2008), que destaca a importância do "ato de contar histórias digitais como ferramenta para ajudar os estudantes a desenvolverem habilidades de alfabetização tecnológica" ${ }^{17}$ (p.23).

Referência semelhante é apontada por Robin ${ }^{10}$ que relaciona o trabalho criativo envolvido na produção das narrativas digitais à promoção do que muitos educadores começaram a chamar de Alfabetização no Século 21, Alfabetização na Era Digital ou Habilidades do Século 21. Independentemente do termo específico utilizado, essas capacidades são descritas como a combinação de: Alfabetização digital - a capacidade de se comunicar com uma comunidade cada vez maior para discutir questões, coletar informações e procurar ajuda; Alfabetização global - a capacidade de ler, interpretar, responder e contextualizar as mensagens a partir de uma perspectiva global; Alfabetização tecnológica - a habilidade de usar computadores e outras tecnologias para melhorar a aprendizagem, produtividade e performance; Alfabetização visual - a habilidade para compreender, produzir e comunicar por meio de imagens visuais; Alfabetização da informação - habilidade para avaliar e sintetizar a informação. Nessa perspectiva, conforme referem Hack e Guedes ${ }^{17}$, a narrativa digital não pode ser vista apenas como "uma técnica para potencializar processos de aprendizagem na educação superior, mas também como uma estratégia de habilitação à literacia digital 
(p.24)". Sob uma perspectiva pedagógica, o desenvolvimento dessas habilidades requer do educando sua participação ativa e crítica no processo educativo. Isso envolve uma prática que considere o seu contexto e promova a sua autonomia, dialogando, desta forma, com as suas necessidades ${ }^{18}$.

A partir dessas considerações sobre a narrativa digital, podemos reforçar a premissa de que a narrativa tradicional, defendida por Bruner ${ }^{3-4}$, como uma possibilidade de produção de conhecimento, com os recursos oferecidos pelas tecnologias, amplia suas possibilidades de produção, estimulando a participação do aluno como sujeito ativo desse processo e promovendo o desenvolvimento de habilidades, como o trabalho colaborativo, o pensamento crítico, entre outros. Além disso, as possibilidades de integração das TDIC em espaços educativos traz à tona uma discussão que persiste na prática educativa, relacionada à necessidade de criar condições para que o aluno possa pensar a sua realidade e trazer as suas experiências para o processo de ensino-aprendizagem. Refere-se a uma educação que considera o contexto do sujeito ${ }^{18}$, por exemplo, trazendo para os espaços de aprendizagem o que eles já utilizam fora deles, como é o caso das diferentes interfaces digitais.

Na educação, as narrativas digitais têm sido utilizadas basicamente em dois contextos distintos: na formação inicial ou continuada de professores e no desenvolvimento de conteúdos curriculares praticamente em todas as áreas do conhecimento ${ }^{1}$. No contexto da educação médica, Sandars e Murray ${ }^{19-20}$ têm apresentado o uso da narrativa digital como meio para estimular a reflexão. Para os autores, este tipo de aprendizagem permite que o indivíduo atribua sentido à sua experiência e, assim, proporciona uma oportunidade para desafiar os pensamentos e sentimentos a respeito de eventos e contribuir na tomada de decisões.

Rodríguez Illera e Monroy ${ }^{2}$ apresentam um estudo de caso realizado com 20 alunos universitários do curso de Comunicação Audiovisual da Universidade de Barcelona, cujo objetivo era analisar a produção das narrativas digitais para identificar e descrever fatores limitantes ou favorecedores que podem influenciar no desenvolvimento de projetos de aprendizagem desta natureza. Os resultados mostraram impactos positivos relacionados ao aumento na motivação dos alunos, estímulo à criatividade e desenvolvimento de competências necessárias nos processos de alfabetização digital. Os efeitos negativos foram algumas limitações relacionadas com determinadas operações técnicas, falta de acesso ao computador, falta de conhecimento ou experiência com a gestão dos recursos de software. Por fim, os autores referem que, em geral, os relatos digitais despertaram um interesse renovado pela narrativa, no sentido teórico e educativo para os investigadores, e pessoal para seus autores, pelas grandes potencialidades que oferecem².

No cenário nacional, Almeida e Valente ${ }^{1}$ também têm apresentado e discutido o uso das narrativas digitais em contextos educativos. Segundo os autores, a presença das TDIC tem propiciado a reconfiguração da prática pedagógica, a abertura e plasticidade do currículo, bem como a coautoria de professores e alunos. No caso das narrativas digitais, além dessas possibilidades, a narrativa passa a ser uma "janela na mente" do aluno, oferecendo ao professor a oportunidade de entender e identificar os conhecimentos do senso comum, as experiências representadas pelos alunos, podendo ajudá-los na compreensão e construção de conhecimento $^{1}$. Os autores apresentam um estudo de caso sobre a produção de narrativas digitais no qual analisaram uma experiência com 12 alunos de mestrado e doutorado da disciplina denominada "Tecnologia e Currículo: fundamentos, políticas, práticas e processos de gestão”, oferecida no segundo semestre de 2012 pela linha de pesquisa de Novas Tecnologias na Educação, do Programa de Pós-Graduação em Educação, que integra o Currículo da Pontifícia Universidade Católica de São Paulo.

Durante o estudo, toda a trajetória de desenvolvimento da disciplina foi registrada na plataforma online do Moodle, e para o desenvolvimento das narrativas digitais, os alunos usaram distintas ferramentas e interfaces (PowerPoint, Word, blog, Prezi, fotolog, Flash) de livre escolha, e com acesso por meio de dispositivos específicos das TDIC, fixos ou móveis. Os autores concluíram que a análise do processo de produção de narrativas digitais evidenciou as contribuições das TDIC no aprendizado da disciplina, especificamente em relação à compreensão dos conceitos em estudo que foram colocados em ação pelos 
alunos e em relação à compreensão do seu universo de conhecimentos, suas experiências, histórias de vida, desejos, valores e caminhos percorridos em sua formação. Por último, apontam a criatividade no desenvolvimento de uma produção hipermídia, proporcionando o desenvolvimento de competências e habilidades de uso dos recursos tecnológicos escolhidos para realizar o trabalho proposto'1.

Quando discutimos o ensino da saúde, devemos considerar que este ainda encontra-se fortemente marcado por uma concepção tradicional, orientado por uma prática pedagógica centrada no professor e marcada pela aquisição de conhecimentos desvinculada da realidade ${ }^{21}$. Nesse modelo, os alunos são vistos como receptores de informações técnico-científicas sobre doenças e tratamentos, em uma perspectiva que desconhece o protagonismo ativo dos aprendizes ${ }^{22}$. Este tipo de formação não prepara os profissionais para trabalhar as dimensões subjetivas, sociais e culturais envolvidas no processo de adoecimento e tratamento dos indivíduos ${ }^{23}$. Além disso, percebe-se por parte do aluno uma dificuldade em relacionar o grande volume de informações teóricas com a realidade dos usuários dos serviços de saúde ${ }^{24}$. Faltam, portanto, estratégias que promovam uma maior participação de todos os atores sociais envolvidos no ensino da saúde, professor, aluno e paciente, de forma ativa e dialógica.

Segundo Batista e Rossit ${ }^{25}$, a formação em saúde é marcada por um ensino onde os conteúdos são trabalhados de forma fragmentada, esperando-se por parte do discente uma visão de totalidade da sua formação profissional. Contudo, os autores questionam esse tipo de ensino que não prepara os profissionais de saúde para serem críticos e reflexivos, uma vez que as situações de aprendizagem na graduação se pautam na transmissão e na repetição dos conteúdos. Não há um processo de construção, mas de acumulação cada vez mais complexa. Defendese, portanto, que a aprendizagem, superando as concepções tradicionais, seja entendida como um processo construtivo e desenvolva um ensino inovador que, entre outras características, privilegie as metodologias que possibilitam interações produtivas, valorizando uma apropriação crítico-reflexiva ${ }^{25}$.

Uma formação que objetive despertar no educando a sua capacidade reflexiva e um olhar ampliado para as suas vivências de aprendizagem é aquela que considera o valor das experiências. Para Dewey ${ }^{26}$, a experiência na prática pedagógica considera o aluno como central no processo de aprendizagem e a sua importância reside na capacidade de promover o aprendizado, uma vez que toda experiência modifica quem a fez e por ela passa. Para tanto, esse caminho requer atividades que promovam uma participação autônoma e criativa do aluno e o desenvolvimento das suas potencialidades. Além disso, deve-se promover uma relação dialética entre teoria e prática ${ }^{27-28}$. No ensino da saúde, quando os alunos estão diante de situações novas, seja nos serviços de saúde, na comunidade ou na prática em si, eles devem ser incentivados a pensar sobre essas experiências e compartilhá-las com o objetivo de atribuir-lhes sentido, relacioná-las com a teoria e direcionar a sua ação futura.

\section{Metodologia}

A presente investigação envolveu a realização de pesquisas acerca da produção acadêmica internacional na base de dados do Portal de Periódicos da Capes, por ser de livre acesso para os pesquisadores a nível nacional e possuir um vasto acervo. Para tanto, foram usados descritores em língua inglesa, seguindo os critérios de busca avançada, somente artigos revisados por pares e sem limitação de período de tempo. A pesquisa foi realizada no primeiro semestre de 2015 utilizando os seguintes descritores: "Digital Storytelling and Health Education", e a segunda usando especificamente "Digital Storytelling and Medical Education". Diante do volume de resultados, alguns critérios para triagem foram estabelecidos a fim de atender ao seguinte objetivo: os artigos deveriam relacionar-se a usos práticos da narrativa digital em contextos educativos no ensino da saúde. Isso implicou a exclusão dos artigos teóricos. Os resultados da busca com os descritores "Digital Storytelling and Health Education" totalizaram 35 artigos, dos quais 31 eram revisados por pares. Em uma primeira leitura dos títulos e resumos, restaram 19 artigos, excluindo-se as repetições 
e aqueles que não estavam relacionados ao objetivo de busca, porém identificou-se que cinco destes não estavam disponíveis e um não contemplava o nosso objetivo inicial, restando, portanto, 13 artigos. Estes foram submetidos a uma leitura completa e análise quanto aos critérios definidos, o que excluiu mais três artigos teóricos e três que não tinham relação com o ensino da saúde. Por fim, foram selecionados para análise sete artigos que apresentavam, após essas etapas, relação com nosso objetivo inicial. O quadro 01 apresenta a distribuição destes artigos por periódico e ano.

Quadro 01. Distribuição dos artigos, por periódico e ano

\begin{tabular}{|c|c|c|c|}
\hline Artigo & Autores & Periódico & Ano \\
\hline $\begin{array}{l}\text { Involving a young person in the development of a } \\
\text { digital resource in nurse Education }\end{array}$ & Fenton, G. & $\begin{array}{l}\text { Nurse Education in } \\
\text { Practice }\end{array}$ & 2014 \\
\hline $\begin{array}{l}\text { The development and evaluation of online stories } \\
\text { to enhance clinical learning experiences across } \\
\text { health professions in rural Australia }\end{array}$ & Paliadelis, P. S. et al & Collegian & 2014 \\
\hline $\begin{array}{l}\text { Promoting Positive Youth Development and } \\
\text { Highlighting Reasons for Living in Northwest } \\
\text { Alaska Through Digital Storytelling }\end{array}$ & Wexler, L. et al. & $\begin{array}{l}\text { Health Promotion } \\
\text { Practice }\end{array}$ & 2013 \\
\hline $\begin{array}{l}\text { Bridging storytelling traditions with digital } \\
\text { technology }\end{array}$ & Cueva, M. et al & $\begin{array}{l}\text { International Journal } \\
\text { Of Circumpolar } \\
\text { Health }\end{array}$ & 2013 \\
\hline $\begin{array}{l}\text { Addressing food insecurity in a Native American } \\
\text { reservation using community-based participatory } \\
\text { research }\end{array}$ & Jernigan, et al. & $\begin{array}{l}\text { Health Education } \\
\text { Research }\end{array}$ & 2012 \\
\hline $\begin{array}{l}\text { Storytelling and professional learning: A } \\
\text { phenomenographic study of students' experience } \\
\text { of patient digital stories in nurse Education }\end{array}$ & Christiansen, A & $\begin{array}{l}\text { Nurse Education } \\
\text { Today }\end{array}$ & 2011 \\
\hline $\begin{array}{l}\text { Challenging the shock of reality through digital } \\
\text { storytelling }\end{array}$ & $\begin{array}{l}\text { Stacey, Gemma; Hardy, } \\
\text { Pip }\end{array}$ & $\begin{array}{l}\text { Nurse Education in } \\
\text { Practice }\end{array}$ & 2011 \\
\hline
\end{tabular}

Fonte: Elaboração dos autores

Os resultados da busca com os descritores "Digital Storytelling" and "Medical Education" totalizaram 16 artigos, sendo que apenas 12 eram revisados por pares. Em uma primeira leitura dos títulos e resumos, restaram oito artigos, excluindo-se as repetições e aqueles que não estavam relacionados ao objetivo de busca (três estavam duplicados e um não fazia referência à narrativa digital). Esses artigos foram submetidos a uma leitura completa e análise quanto aos critérios definidos, o que excluiu dois artigos que também apareceram na busca com os descritores "Digital Storytelling" and "Health Education", um editorial, dois artigos teóricos e um que tratava sobre um projeto de trabalho com a narrativa, portanto, algo que ainda seria desenvolvido. Dessa forma, restaram apenas dois artigos que apresentavam e discutiam o uso da narrativa digital na educação médica, conforme apresentado no quadro 02. 
Quadro 02. Distribuição dos artigos, por periódico e ano

\begin{tabular}{l|l|l|l}
\hline Artigo & Autores & Periódico & Ano \\
\hline $\begin{array}{l}\text { Digital storytelling for reflection in } \\
\text { undergraduate medical education: A pilot } \\
\text { study }\end{array}$ & Sandars, J.; Murray, C. & $\begin{array}{l}\text { Education for Primary } \\
\text { Care }\end{array}$ & 2009 \\
\hline $\begin{array}{l}\text { A pediatric digital storytelling system for third } \\
\text { year medical students: The virtual pediatric } \\
\text { patients }\end{array}$ & $\begin{array}{l}\text { D'Alessandro, D.M.; Lewis, } \\
\text { T.E.; D'Alessandro, M.P. }\end{array}$ & $\begin{array}{l}\text { BMC Medical } \\
\text { Education }\end{array}$ & 2004 \\
\hline
\end{tabular}

Fonte: Elaboração dos autores

Após a leitura completa de todos os artigos, estes foram organizados quanto à proposta de uso da narrativa digital em contextos de ensino da saúde. Nesse sentido, identificamos duas perspectivas principais de práticas pedagógicas, que orientaram a sua apresentação nos resultados. A primeira traz a narrativa como processo de reflexão do sujeito, seja ele o aluno, o profissional de saúde ou o paciente. E a segunda envolve a narrativa como recurso para as práticas de ensino, como por exemplo, o uso de narrativas de pacientes em ambientes na internet para que os alunos possam aprender com elas. A partir desse entendimento, apresentamos os principais aspectos que respondiam ao nosso objetivo, em relação às práticas pedagógicas.

\section{Resultados e discussões}

\section{Narrativas digitais em contextos educativos no ensino da saúde}

A produção acadêmica sobre o tema tem apresentado artigos com diferentes perspectivas, envolvendo práticas pedagógicas em situações bem particulares, tais como a criação de narrativas pelos próprios alunos como forma de promover a reflexão para a aprendizagem, a sua produção por profissionais de saúde, e a valorização das narrativas de pacientes, disponibilizadas na internet como recurso para aproximar os alunos desse universo particular relacionado ao adoecimento do indivíduo.

Na pesquisa realizada com os descritores "Digital Storytelling and Health Education", a primeira perspectiva (a narrativa como processo de reflexão do sujeito) foi observada nos seguintes artigos: Cueva et al..$^{29}$, Stacey e Hardy ${ }^{30}$ e Wexler et al..$^{31}$. Os demais artigos se relacionaram ao uso da narrativa como um recurso para as práticas de ensino: Jernigan et al. ${ }^{32}$, Fenton ${ }^{33}$, Paliadelis et al. ${ }^{34}$ e Christiansen ${ }^{35}$. Na pesquisa com os descritores "Digital Storytelling" and "Medical Education", cada artigo se relacionou a uma perspectiva de abordagem. O trabalho de Sandars e Murray ${ }^{20}$ foi caracterizado a partir do uso da narrativa para a reflexão do aluno. E o outro artigo, D’Alessandro, Lewis e D'Alessandro ${ }^{36}$, envolveu o uso das narrativas em simuladores na internet.

Cueva et al. ${ }^{29}$ apresentam o processo de criação de narrativas digitais por Agentes Comunitários de Saúde (ACS) do Alasca com a finalidade de compartilhar informações de saúde relacionadas ao câncer. Com a criação de uma história pessoal digital, os participantes integraram seu conhecimento sobre o câncer com a sua experiência pessoal para refletir criticamente sobre a mensagem de saúde que queriam compartilhar dentro de sua rede de relacionamentos. Consequentemente, os participantes descreveram como a criação de narrativas aumentou seu conhecimento sobre o câncer, exigindo-lhes avaliar, sintetizar e aplicar seus conhecimentos em uma narrativa digital. Os autores do artigo reforçam a importância da inclusão do pensamento e da expressão dos alunos como base para o desenvolvimento da compreensão crítica e da experiência pessoal. Dessa forma, a combinação da narrativa digital com a educação sobre o câncer feita 
pelos Agentes Comunitários de Saúde cria possibilidades para promover a aprendizagem, uma vez que valoriza a experiência e a cultura de cada participante. Sob a perspectiva dos agentes de saúde, a produção de narrativas digitais foi vista com entusiasmo, pois puderam aprender a usar a tecnologia baseada em computador para criar e contar uma história pessoal, enfatizando a acessibilidade das narrativas digitais até mesmo para aqueles com pouco conhecimento sobre as tecnologias ${ }^{29}$.

Stacey e Hardy ${ }^{30}$ apresentam um trabalho relacionado à criação de narrativas digitais reflexivas de enfermeiros recém-formados sobre eventos nos quais encontraram dificuldades durante a transição de estudante a profissional de enfermagem. Os autores usam o conceito de 'reality shock' (choque de realidade) referente à reação dos novos profissionais quando eles se encontram em uma situação de trabalho para a qual passaram vários anos se preparando e achavam que estavam prontos e, de repente, descobrem que não estão. Os autores defendem o aprendizado a partir da reflexão que o indivíduo faz da sua experiência, dialogando, portanto, com o pensamento de Dewey (1938). No estudo, oito enfermeiros recém-formados foram convidados a refletir sobre as experiências recentes e expectativas em relação à prática profissional com a criação de suas histórias digitais. Os resultados deste estudo sugerem que as narrativas digitais possibilitam aos alunos assumirem o papel de contadores de histórias e compartilharem suas experiências em relação às expectativas com a prática profissional ${ }^{30}$.

Wexler et al..$^{31}$ tratam sobre a produção de narrativas digitais por adolescentes como estratégia para prevenção do suicídio, em um programa chamado 'Positive Youth Development', no nordeste do Alasca. Para ajudar os jovens em seus processos de desenvolvimento, a narrativa digital pode ser um recurso para que eles representem sua cultura e identidade, para destacar os aspectos positivos de suas vidas e fortalecer as conexões com as pessoas importantes para eles. Baseados na literatura corrente sobre a narrativa digital, os autores enfatizam o uso das ferramentas de mídia visual como forma de promover o engajamento da juventude com a sociedade. As histórias digitais, quando compartilhadas com os membros de uma comunidade, podem incentivar a transmissão de conhecimentos relevantes e estabelecer um espaço para a resolução de problemas coletivos. Os autores concluem que o projeto da narrativa digital ofereceu aos jovens uma forma de adquirir senso de domínio e realização pessoal, destacando aspectos positivos de si mesmos, suas vidas e suas razões de viver. Assim, a narrativa digital apresenta uma abordagem promissora para a promoção da saúde, que pode ser usada para reforçar as identidades culturais necessárias para oferecer aos jovens uma vida adulta saudável ${ }^{31}$.

Jernigan et al..$^{32}$ usaram a narrativa digital para adaptar uma ferramenta de avaliação das causas de insegurança alimentar em uma comunidade indígena. Essa ferramenta é conhecida como 'Tool for Health and Resilience in Vulnerable Environments' (THRIVE). Guiados por uma orientação de pesquisa participativa baseada na comunidade, a ferramenta foi adaptada utilizando a narrativa digital e implementada em uma série de grupos focais. O objetivo de uso das histórias digitais foi acompanhar cada fator da ferramenta THRIVE, ilustrando seu significado e sua manifestação no contexto local. Para os autores, a inclusão da narrativa digital para adaptar a ferramenta foi essencial para o seu uso na avaliação das causas da insegurança alimentar em uma determinada comunidade indígena ${ }^{32}$. Apesar de não apresentar a forma como ela foi usada e explorar melhor os benefícios da narrativa digital, este estudo mostra outra perspectiva de trabalho que pode ser discutida em contextos educativos, como a possibilidade de redesenhar práticas ou estratégias de trabalho a partir da contribuição das tecnologias digitais.

Fenton $^{33}$ apresenta o desenvolvimento e avaliação de um recurso digital, um DLO (digital learning object) na formação do enfermeiro. O DLO é uma atividade digital que pode ser integrada em programas educacionais. Nesse estudo, o objetivo do projeto foi desenvolver e incorporar um DLO aos módulos ensinados, a fim de expor os alunos à experiência vivida de uma pessoa jovem com uma doença potencialmente fatal, a partir da sua narrativa, e avaliar as percepções dos alunos sobre esta ferramenta como estratégia de ensino e aprendizagem. Os autores defendem a importância de valorizar a experiência 
dos pacientes e aproximá-los, assim, dos alunos, incentivando uma prática mais sensível e humanizada. Os participantes do estudo destacaram o valor de ouvir a história do paciente, permitindo obter conhecimento e desenvolver empatia com a sua experiência com uma doença potencialmente fatal. A maioria dos alunos afirmou que o DLO foi útil, de fácil acesso, principalmente por integrar texto, som e recursos visuais.

O estudo de Paliadelis et al. ${ }^{34}$ descreve o desenvolvimento e a avaliação de histórias on-line para melhorar a experiência de aprendizagem clínica nas profissões de saúde em uma área rural da Austrália. A produção das narrativas digitais aconteceu sob outra perspectiva. Estas não foram produzidas pelos próprios alunos, mas por um grupo de profissionais, docentes e clínicos, com a finalidade de abranger questões de interesse para os alunos. O projeto foi concebido para apoiar o desenvolvimento profissional dos estudantes e seus supervisores clínicos, oferecendo um programa de e-learning interprofissional e inovador, que, por meio de um modelo de narração de histórias, contribuiria para experiências de aprendizagem eficazes integradas no trabalho. Uma vez que os alunos tiveram acesso às histórias, eles foram incentivados a refletir sobre o que tinham aprendido. Os resultados indicaram que os alunos avaliaram positivamente o uso das histórias on-line e valorizaram a atividade de contar histórias para a aprendizagem e como meio para promover a "conexão" emocional entre personagens e conteúdo, promovendo a reflexão acerca das experiências com as quais tiveram acesso. Os resultados confirmaram trabalhos anteriores, indicando a contribuição das histórias na aprendizagem mediada por fatores como o envolvimento emocional, além de estímulo à reflexão ${ }^{34}$.

O trabalho de Christiansen ${ }^{35}$ reforça o potencial da narrativa para a aprendizagem profissional. A acessibilidade das novas tecnologias e o uso de oficinas de contar histórias digitais têm apoiado o desenvolvimento de grandes arquivos de narrativas digitais baseadas na web, cada vez mais utilizadas no âmbito da educação profissional para apoiar a aprendizagem dos alunos. O estudo utilizou uma abordagem fenomenográfica para identificar e compreender as diferentes formas em que os alunos do terceiro ano de um curso de graduação em Enfermagem experimentaram e deram sentido a histórias digitais dos pacientes. Tais histórias serviram para estimular o debate entre os estudantes. De acordo com a autora, por meio de um processo de construção de significados, de engajamento e reflexão emocionais, os alunos puderam gerar novas percepções que têm o potencial de transformar o desenvolvimento de sua identidade profissional. As narrativas de pacientes, por exemplo, representam uma relevante forma de expor os alunos à experiência de adoecimento, incentivando uma prática mais humanizada ${ }^{35}$.

Os artigos que resultaram da pesquisa no contexto da educação médica, apesar de serem apenas dois, sinalizam um campo de estudo e trabalho que tem se ampliado nessa formação, no sentido de buscar caminhos que favoreçam a participação ativa do sujeito educando e a criação de espaços de reflexão no processo educativo. Os casos apresentados referem-se especificamente aos usos da narrativa digital no ensino médico a partir de duas abordagens: o primeiro caso discute a produção de narrativas digitais pelos próprios alunos como forma de estimular a reflexão para a aprendizagem ${ }^{20}$; no segundo artigo, são valorizadas as histórias de pacientes disponibilizadas na internet como recurso para aproximar os alunos desse universo particular relacionado ao adoecimento do indivíduo ${ }^{36}$.

A partir de um estudo piloto com um grupo de 12 alunos, Sandars e Murray ${ }^{20}$ testaram a hipótese de que a narrativa digital tem o potencial de oferecer uma abordagem inovadora para propiciar a reflexão dos estudantes do primeiro ano da graduação. Os alunos usaram a narrativa digital como exercício de aprendizagem reflexiva no módulo de desenvolvimento pessoal e profissional, cujo objetivo foi estimular a reflexão sobre suas próprias experiências a partir do primeiro encontro com o paciente em sua casa,. Os estudantes receberam informações sobre como criar uma narrativa digital e foram encorajados a usar seus celulares para coletar imagens que capturassem um pensamento ou sentimento particular relacionado à sua experiência do encontro com o paciente. A partir dessas imagens, os alunos criaram a narrativa digital usando o PowerPoint, uma vez que este era um recurso familiar a todos. Os resultados revelaram que os alunos se envolveram com entusiasmo e foram criativos no processo de produção de sua narrativa digital. 
Este foi considerado um aspecto contrastante em relação às metodologias geralmente utilizadas no seu curso de graduação e que não envolvem os alunos nessa atividade reflexiva. Para os autores, o processo de criação da narrativa digital estimulou os alunos a refletir sobre porque haviam coletado, selecionado e apresentado as várias fotografias. Acreditam que todo o processo, desde a criação da narrativa até a sua apresentação, provocou nos alunos vários 'disorientating dilemmas' (dilemas desorientadores), uma espécie de gatilho para estimular a reflexão, ou seja, os levaram a pensar sobre cada evento particular e como buscar alternativas para resolvê-lo ou repensá-lo ${ }^{20}$.

D’Alessandro, Lewis e D’Alessandro ${ }^{36}$ apresentam uma proposta de trabalho com o uso das narrativas digitais integradas a simuladores de pacientes baseados em computador. Para os autores, o uso destas simulações é comum para orientar o aprendizado de estudantes de medicina. A partir de entrevistas realizadas com pacientes e familiares que vivenciaram problemas pediátricos, foi criado um modelo de narrativa digital para ser disponibilizado em simuladores da internet. Foram criadas oito histórias digitais para este sistema de narrativa, apresentando alguns problemas mais recorrentes nas crianças, como febre, dores nas pernas, na garganta, vômitos e diarreia. Os autores acreditam ter cumprido a maior parte das metas educacionais para as quais o sistema de narrativa digital foi desenvolvido, uma vez que as palavras do próprio paciente foram usadas para descrever o problema clínico, enfatizado juntamente com a apresentação do processo da doença, acrescentado de uma discussão no final da história. Como conclusão do estudo, ressaltam que o sistema de narrativa digital integrado aos simuladores são meios mais rápidos e de fácil acesso para os alunos. Em relação ao potencial das narrativas, destacam a arte de contar histórias na medicina como um importante método de valores e atitudes de ensino, relevantes para o desenvolvimento profissional ${ }^{36}$.

Os trabalhos se relacionam a práticas pedagógicas, em geral, no ensino da enfermagem e da medicina, apresentando experiências de uso no processo de ensino-aprendizagem, seja com os alunos em formação ou com os profissionais de saúde. Sobre esse aspecto, destaca-se a sua utilização na prática dos profissionais de saúde, como é apresentado por Cueva et al. ${ }^{29}$. Neste estudo, a produção da narrativa digital pelos agentes de saúde revela o seu potencial como forma de valorizar a experiência individual destes profissionais e promover o compartilhamento de experiências que contribuem para a formação continuada em saúde e para a construção de conhecimentos sobre determinado tema.

Em relação à realização de atividades de produção da narrativa pelos alunos, os estudos compartilham a observação de que a narrativa digital possibilita a promoção da criatividade, criticidade e reflexão do estudante, além de permitir o compartilhamento de suas experiências ${ }^{20,30,31,33}$. A outra perspectiva de trabalho apresentada revela a importância de usar as narrativas dos pacientes para o aprendizado do aluno, destacando a possibilidade de conhecer melhor esse sujeito de cuidado e suas histórias de vida e adoecimento, permitindo assim um atendimento mais individualizado e um olhar ampliado sobre o mesmo ${ }^{34-36}$. A narrativa do paciente permite compreendê-lo além da sua enfermidade. E configura-se como elemento indispensável para uma formação humanística, para a abordagem holística dos fenômenos saúde-doença ${ }^{37}$.

No ensino da saúde, a adoção de práticas pedagógicas que valorizem o aprendizado a partir da narrativa do outro no processo ensino-aprendizagem permite o acesso a dimensões que ultrapassam o plano biológico e consideram os aspectos cognitivos, sociais e culturais. Essa particularidade refere-se em grande parte ao fato de a narrativa permitir que a experiência do sujeito seja significada para si e para os outros ${ }^{8}$. Dessa forma, olhar para a mesma situação é muito peculiar e varia de acordo com a posição que o sujeito ocupa, de onde ele fala. Isso é relevante para pensar, por exemplo, a compreensão do aluno sobre sua experiência, seja na relação profissional de saúde-paciente ou aluno-docente, e dos significados construídos e reconstruídos a partir dela.

Aliadas à função da narrativa no ato de educar, as TDIC criam possibilidades de transformação nos processos educativos e, consequentemente, no ensino da saúde, em todos os níveis, influenciando os conceitos e processos de construção do conhecimento e as metodologias de ensino-aprendizagem ${ }^{38}$. 
Desta forma, podem ser trabalhadas em contextos que favoreçam a realização de projetos e atividades significativas, nos quais o conhecimento possa ser construído e reconstruído, possibilitando, formas diversas de participar do processo educativo.

\section{Considerações Finais}

A narrativa digital configura-se como um conceito em evolução ${ }^{11}$. No entanto, tem sido apresentada e discutida quanto a sua contribuição na educação, em diferentes áreas, revelando assim suas possibilidades de trabalho e aplicação. Seu interesse educativo encontra-se na capacidade de contar uma experiência e refletir sobre ela, dando sentido aos acontecimentos, característica peculiar da narrativa e, com o aporte das mídias digitais, amplia as formas de representação a partir de diferentes linguagens. Nesse trabalho, a discussão sobre a narrativa digital no ensino da saúde é revelada a partir de diferentes práticas pedagógicas, sugerindo, dessa forma, caminhos para sua integração na educação. Destaca-se a discussão em torno da sua contribuição para a aprendizagem do aluno ou do profissional de saúde, a visibilidade oferecida ao paciente, revelando suas histórias de vida e adoecimento, bem como a sua função relacionada ao letramento digital.

A partir dessas considerações sobre a narrativa digital no ensino da saúde, propostas neste artigo, discute-se quanto à sua contribuição para uma prática pedagógica que valorize a participação ativa tanto dos educadores quanto dos educandos, permitindo um movimento dialógico no processo de ensinoaprendizagem e novas formas de pensar a educação e o papel dos sujeitos envolvidos. Nesse sentido, a narrativa digital pode ser trabalhada em outras áreas de conhecimento, com a finalidade de transformar os processos pedagógicos mediados pelas tecnologias digitais e pela valorização da experiência dos sujeitos. Não podemos desconsiderar que a narrativa digital, quando trabalhada nessa perspectiva, adquire um interesse educativo, uma vez que se configura como um meio para o aluno dar sentido ao que faz, além de favorecer a sua motivação em relação ao aprendizado² .

Os resultados apresentados demonstram que a produção sobre o tema ainda é incipiente, uma vez que restaram poucos artigos relacionados aos usos práticos da narrativa digital nesses contextos de ensino da saúde. Além disso, a maioria deles é de publicação recente, de menos de cinco anos. No entanto, há uma quantidade relevante de artigos teóricos, sugerindo um maior debate sobre o tema e caminhos para discutilo nos contextos educativos. Dessa forma, a presente discussão pretende ampliar o olhar para o ensino da saúde a partir de uma perspectiva transformadora, que encontra nas narrativas digitais possibilidades para alcançar uma prática pedagógica mais participativa e reflexiva. Entendemos que o debate sobre o tema não se limita a esse artigo, mas representa um ponto de partida para orientar estudos futuros sobre a integração das narrativas digitais em contextos educativos, em particular, no ensino da saúde.

\section{Referências}

1. Almeida MEB, Valente, JA. Integração currículo e tecnologias e a produção de narrativas digitais. Currículo sem Fronteiras. 2012;12(3):57-82.

2. Rodríguez Illera, JL, Londoño Monroy G. Los relatos digitales y su interés educativo. Educação, Formação \& Tecnologias. 2009;2(1):5-18.

3. Bruner J. The narrative construction of reality. Critical Inquiry. 1991;17:1-21.

4. $\quad$ Bruner J. Cultura da Educação. Edições 70; 1996.

5. Gomes MPC, Ribeiro VMB, Monteiro DM, Leher EMT, Louzada RC. O uso de metodologias ativas no ensino de graduação nas ciências sociais e da saúde - avaliação dos estudantes. Ciência \& Educação. 2010;16(1):181-198.

6. Mitre SM, Siqueira-Batista R, Girardi-De-Mendonça JM, Morais-Pinto NM., Meirelles CAB, Pinto-Porto C et al. Metodologias ativas de ensino-aprendizagem na formação profissional em saúde: debates atuais. Ciência \& Saúde Coletiva. 2008;13(2):2133-2144. 
7. Cunha MI. Conta-me Agora! as narrativas como alternativas pedagógicas na pesquisa e no ensino. Rev. Fac. Educ. 1997;23(1-2).

8. Galvão C.Narrativas em Educação. Ciência \& Educação. 2005;11(2):327-345.

9. Robin B. The educational uses of digital storytelling. [ebook] University of Houston: Educational Uses of Digital Storytelling Website; 2005.

10. Robin BR. Digital storytelling: a powerful technology tool for the 21st century classroom. Theory Into Practice. 2008;47(3):220-228.

11. Jesus AG. Narrativa digital: uma abordagem multimodal na aprendizagem de inglês. (Dissertação de Mestrado), Braga: Universidade do Minho; 2010.

12. Hull GA, Nelson ME. Locating the Semiotic Power of multimodality. Written Communication. $2005 ; 22(2) ; 224-261$.

13. Figueiredo JCT. Digital Storytelling no eLearning: estudo de caso da sua aplicação a um módulo no ensino superior. (Dissertação de Mestrado). Lisboa: Universidade Aberta; 2014.

14. Lourenço C, Ramos A. Integrating multiple literacies and digital storytelling in language learning: a case study. Apresentado em: International Conference "ICT for Language Learning, 5th edition; 2012.

15. Joly M. Introdução à Análise da Imagem. Lisboa: Edições 70; 1994.

16. Leeds Metropolitan University. Digital approaches to academic reflection a digital storytelling study guide. 2012

17. Hack JR, Guedes O. Digital Storytelling, Educação Superior e Literacia Digital. Roteiro. 2013;38(1);9-32.

18. Freire P. Pedagogia da autonomia: saberes necessários à prática educativa. São Paulo: Paz e Terra; 1996,

19. Sandars J, Murray C, McPherson M. Reflective learning for the Net Generation of students: the digital storytelling approach. 2008 Retrieved from: http://www.lts.leeds.ac.uk/bulletin/issue18/page9.php.

20. Sandars J, Murray C Digital storytelling for reflection in undergraduate medical education: a pilot study. Educ Prim Care. 2009 Nov;20(6):441-4.

21. González AD, Almeida MJ. Integralidade da saúde: norteando mudanças na graduação dos novos profissionais. Ciênc. saúde coletiva. 2010;15(3):757-762.

22. Ceccim RB, Feuerwerker LCM. Mudança na graduação das profissões de saúde sob o eixo da integralidade. Cad. Saúde Pública. 2004;20(5):1400-1410.

23. Ribeiro VMB Discutindo o conceito de inovação curricular na formação dos profissionais de saúde: o longo caminho para as transformações no ensino médico. Trabalho, Educação e Saúde. 2005;3(1):91-121.

24. Cezar PHN. Transição paradigmática na educação médica: um olhar construtivista dirigido à aprendizagem baseada em problemas. Rev. bras. educ. med. 2010; 34(2):298-303.

25. Batista $\mathrm{SH}$, Rossit RA. Aprendizagem, ensino e formação em saúde: das experiências às teorias em construção. In: Batista, NA, Batista SH, organizadores. Docência em saúde: temas e experiências. 2a ed. rev. amp. São Paulo: Editora Sena;, 2014. p. 51-68.

26. Dewey J. Experiência e Educação. São Paulo: Editora Nacional; 1996.

27. Freinet C Pedagogia do Bom Senso. São Paulo: Martins Fontes; 2004.

28. Amorim GCC, Castro AMN, Silva MFS. Teorias e práticas pedagógicas de Cèlestin Freinet e Paulo Freire. In: Anais do IV FIPED - Fórum Internacional de Pedagogia. Campina Grande, REALIZE Editora; 2012.

29. Cueva M, Kuhnley R., Revels, LJ, Cueva K, Dignan M, Lanier AP. Bridging storytelling traditions with digital technology. Int J Circumpolar Health. 2013;72.

30. Stacey G, Hardy P. Challenging the shock of reality through digital storytelling. Nurse Educ Pract. 2011 Mar;11(2):159-64

31. Wexler L, Gubrium A, Griffin M, DiFulvio G. Promoting positive youth development and highlighting reasons for living in Northwest Alaska through digital storytelling. Health Promot Pract. 2013;14(4):617-623.

32. Jernigan BBV, Salvatore AL, Styne DM, Winkleby M. Addressing food insecurity in a Native American reservation using community-based participatory research. Health Educ Res. 2012;27(4):645-55. 
33. Fenton G. Involving a young person in the development of a digital resource in nurse Education. Nurse Educ Pract. 2014;14(1):49-54

34. Paliadelis PS, Stupans I, Parker V, Piper D, Gillan P, Lea J et al. The development and evaluation of online stories to enhance clinical learning experiences across health professions in rural Australia. Collegian. 2015;22(4):397-403.

35. Christiansen A. Storytelling and professional learning: A phenomenographic study of students' experience of patient digital stories in nurse Education. Nurse Educ Today. 2011;31(3):289-93.

36. D'alessandro DM, Lewis TE, D'alessandro MP. A pediatric digital storytelling system for third year medical students: The virtual pediatric patients. BMC Medical Education, 2004. 4(10).

37. Canesqui AM. organizador. Olhares socioantropológicos sobre os adoecidos crônicos. São Paulo: Hucitec; Fapesp; 2007

38. Schall, V. T.; Modena, C. M As novas tecnologias de informação e comunicação em educação em saúde. In: Minayo, M. C. S.; Coimbra júnior, C. E. A. Críticas e atuantes: ciências sociais e humanas em saúde na América Latina. Rio de Janeiro: Fiocruz, 2005, p. 245-255. 